\title{
Mucinous Cystadenoma of the Appendix Mimicking Polycystic Kidney Disease
}

Keina Nozaki, Yoshifumi Ubara, Yuhji Marui, Shinji Tomikawa

Nephrology Center, Toranomon Hospital

A 70-year-old Japanese male with autosomal dominant polycystic kidney disease (ADPKD) and chronic renal failure presented with abdominal distention. His family history was remarkable for ADPKD in his mother, a brother and a sister. He had been receiving hemodialysis for 10 years and his general health had otherwise been maintained.

Computed tomography (CT) in the axial and coronal views showed both kidneys to have been almost completely replaced by multiple cysts and both occupied considerable space in the abdomen, consistent with ADPKD (Figure-1). It was also revealed that the largest cyst, which measured $160 \mathrm{~mm}$ in diameter, was located at the lower pole of the right kidney.

Renal transcatheter arterial embolization was performed for both kidneys to achieve volume reduction because abdominal distention usually results from progressive dilatation of renal cysts and is therefore one of the most common symptoms of ADPKD. Transcatheter arterial embolization is standard treatment for ADPKD at our hospital. Needle aspiration of the largest cyst was performed at the same time, and the cytological result was benign (Class II).

Two years later, the patient presented again with progressive abdominal distention and pain.

CT two years after embolization showed that only the largest cyst on the right side was refractory to treatment and had increased in size to $280 \mathrm{~mm}$ in diameter, while the other cysts had shrunk (Figure-2).
Laparotomy was subsequently performed. During exploration of the abdominal cavity, the largest cyst, which was believed to be one of the renal cysts, was identified as being completely separate from the aggregation of other cysts, instead being contiguous with the appendix. We performed an appendectomy together with resection of the largest cyst and right nephrectomy. Histological examination demonstrated mucinous cystadenoma of the appendix. The postoperative course was uneventful. The patient has been closely followed up for one year without relapse.

Mucinous cystadenoma is accounting for more than half of appendiceal mucoceles, cystic neoplasms characterized by distension of the appendiceal lumen with mucus. As histolgical subtypes, appendiceal mucoceles also include retention cysts, mucosal hyperplasia, and cystadenocarcinoma (1-4).

CT scan or other imaging studies can lead to a presumptive diagnosis of an appendiceal mucocele. However, the differential diagnosis can be challenging and should include cysts of other origins, especially, as in our case, when CT scan or ultrasonography is controversial, but fails to demonstrate the continuity of the lesion with the cecum $(5,6)$.

To our knowledge, this is one of the largest reported cases of mucinous cystadenoma of the appendix and also the first case associated with ADPKD. There is a case of appendiceal mucinous cystadenoma of the same size as this case, which caused bilateral hydronephrosis and 
Figure 1 - Contrast-enhanced CT of the abdomen before embolization reveals bilateral polycystic kidneys, with the largest cyst (arrow) occupying the right lower quadrant of the abdomen. A-C) Axial views at different levels. D) Coronal view.
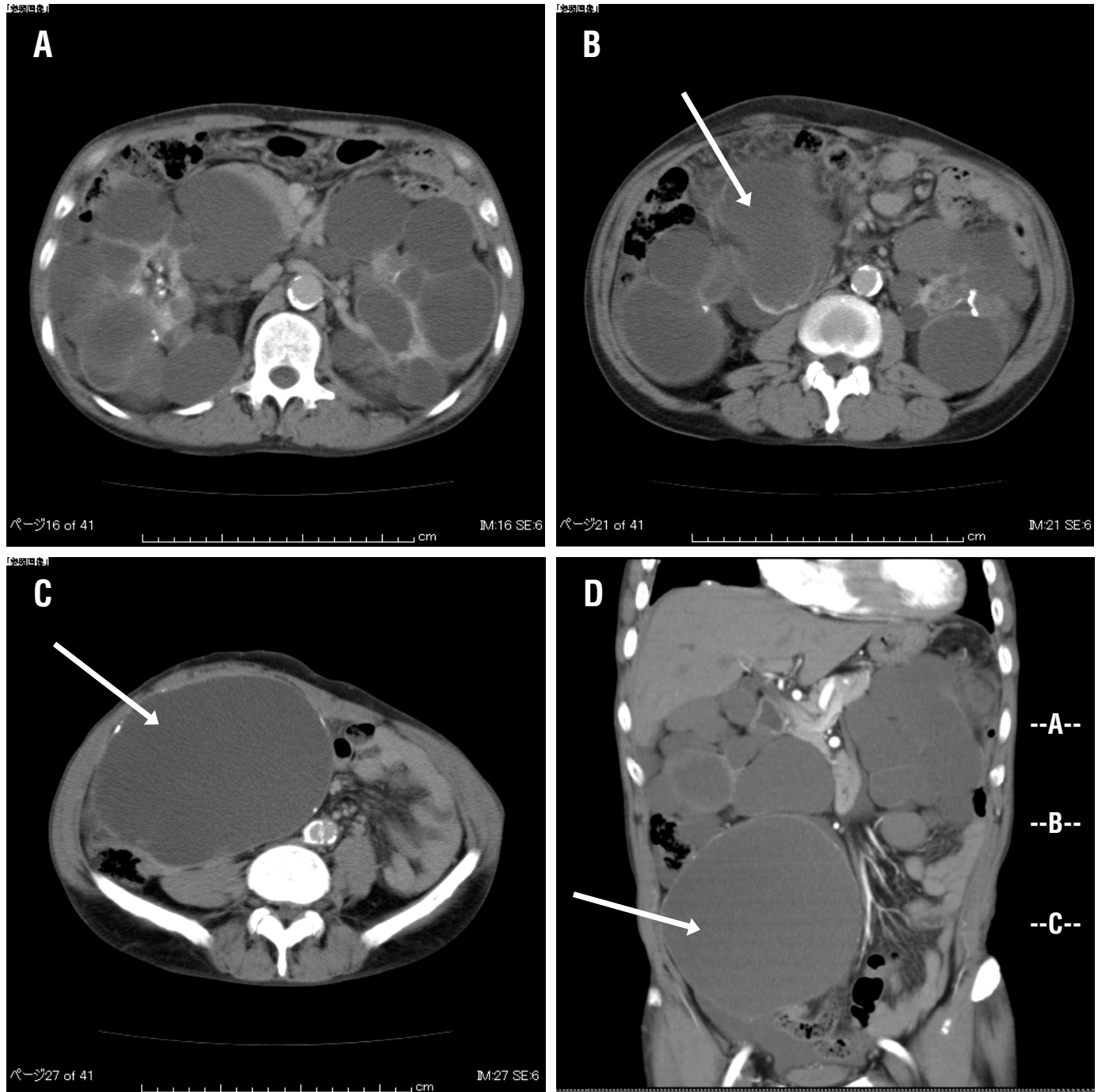

renal failure (7). There are several other reports of mucinous cystadenoma of the appendix misdiagnosed as a cyst of another origin, such as liver cyst, renal cyst and right adnexal cyst (8-12). This case demonstrates that mucinous cystadenoma of the appendix and other cystic lesions should be considered in the differential diagnosis of ADPKD.

\section{ABBREVIATIONS}

ADPKD: autosomal dominant polycystic kidney disease

CT: Computed tomography 
Figure 2 - Contrast-enhanced CT of the abdomen two years after embolization demonstrates, in comparison with Figure 1 , that only the largest cyst had increased in size while the others had regressed.

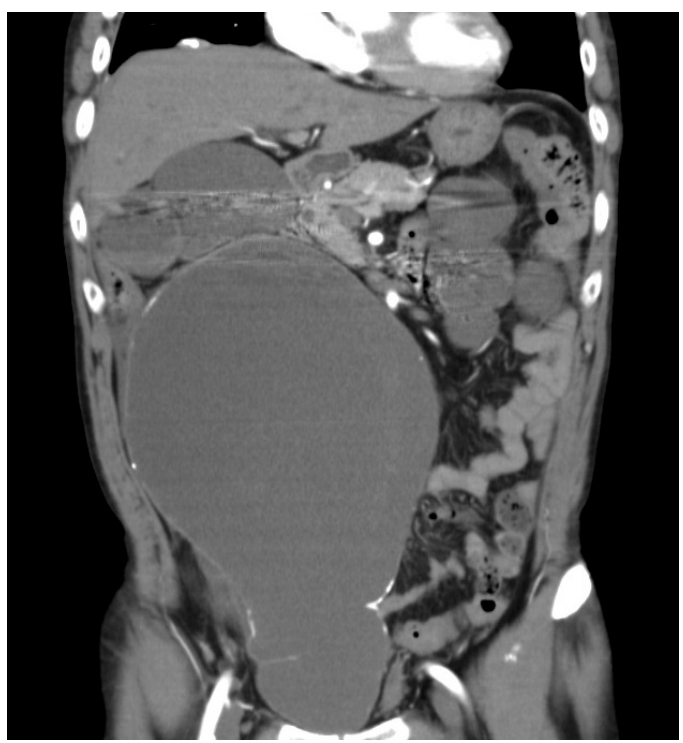

\section{REFERENCES}

1. Higa E, Rosai J, Pizzimbono CA, Wise L: Mucosal hyperplasia, mucinous cystadenoma, and mucinous cystadenocarcinoma of the appendix. A re-evaluation of appendiceal "mucocele". Cancer. 1973; 32: 1525-41.

2. Landen S, Bertrand C, Maddern GJ, Herman D, Pourbaix A, de Neve A, et al.: Appendiceal mucoceles and pseudomyxoma peritonei. Surg Gynecol Obstet. 1992; 175: 401-4.

3. Qizilbash AH: Mucoceles of the appendix. Their relationship to hyperplastic polyps, mucinous cystadenomas, and cystadenocarcinomas. Arch Pathol. 1975; 99: 548-55.
4. Isaacs KL, Warshauer DM: Mucocele of the appendix: computed tomographic, endoscopic, and pathologic correlation. Am J Gastroenterol. 1992; 87: 787-9.

5. Madwed D, Mindelzun R, Jeffrey RB Jr: Mucocele of the appendix: imaging findings. AJR Am J Roentgenol. 1992; 159: 69-72.

6. Koktener A, Akin K, Kosehan D, Dener C: Primary appendicealtumors: clinical imaging and pathological findings. Report of four cases. JBR-BTR. 2011; 94: 63-5.

7. Ilbeigi P, Lombardo S, Sadeghi-Nejad H: Unusual cause of obstructive uropathy. Int Urol Nephrol. 2005; 37: 505-6.

8. Balci 0, Ozdemir S, Mahmoud AS: Appendiceal mucocele mimicking a cystic right adnexal mass. Taiwan J Obstet Gynecol. 2009; 48: 412-4.

9. Scaffa C, Di Bella 0, Tartaglia E, Rotondi M, Lup F, Messalli EM: Surgical approach to appendiceal mucocele mimicking an adnexal complex mass: case report. Eur J Gynaecol Oncol. 2007; 28: 503-5.

10. Kalu E, Croucher C: Appendiceal mucocele: a rare differential diagnosis of a cystic right adnexal mass. Arch Gynecol Obstet. 2005; 271: 86-8.

11. Parsons JK, Freeswick PD, Jarrett TW: Appendiceal cystadenoma mimicking a cystic renal mass. Urology. 2004; 63: 981-2.

12. Krieg A, Esch JS 2nd, Poll LW, Braunstein S, Knoefel WT: Mucinous cystadenoma of the appendix misdiagnosed as cystic hydatid disease of the liver: a case report. J Med Case Reports. 2008; 2: 218.

Correspondence address: Dr. Keina Nozaki Toranomon Hospital,

Kajigaya 1-3-1, Takatsu-ku, Kawasaki, Kanagawa, 213-8587, Japan Fax: + 81 44-877-5333 E-mail keina.nozaki@gmail.com 